\title{
Convergent Total Synthesis of (+)-Calcipotriol: A Scalable, Modular Approach to Vitamin D Analogs
}

Authors: Jieyu Gu ${ }^{\S \dagger}$, Kevin X. Rodriguez ${ }^{\S \dagger}$, Yuzuru Kanda ${ }^{\dagger}$, Shenghua Yang ${ }^{\dagger}$, Michal Ociepa ${ }^{\dagger}$, Henrik Wilke $^{\dagger}$, Arteen V. Abrishami ${ }^{\dagger}$, Lars Jørgensen ${ }^{\perp}$, Tine Skak-Nielsen ${ }^{\perp}$, Jason S. Chen ${ }^{\dagger}$, Phil S. Baran ${ }^{* \dagger}$

\section{Affiliations:}

${ }^{\dagger}$ Department of Chemistry, The Scripps Research Institute, 10550 North Torrey Pines Road, La Jolla, CA 92037, USA.

${ }^{\perp}$ LEO Pharma A/S, Industriparken 55, 2750 Ballerup, Denmark USA.

${ }^{*}$ Correspondence to: pbaran@scripps.edu

\begin{abstract}
:
Vitamin D is a group of seco-steroids with diverse bioactivities. An enormous amount of effort was expended by medicinal chemists to search for Vitamin D analogs that could exhibit prodifferentiating and antiproliferative effects on normal and cancer cells as well as immunomodulatory effects without causing hypercalcemia. A convergent approach for the total synthesis of calcipotriol (brand name: Dovonex), a proven Vitamin D analog used for the treatment of psoriasis, and medicinally relevant synthetic analogs is described. Given the rich synthetic history of the Vitamin D family, a complete novel approach towards both the A-ring and CD-ring is reported. From a retrosynthetic standpoint, hidden symmetry within the decorated A-ring is disclosed, which allowed for scalable quantities of this advanced intermediate. In addition, a radical retrosynthetic approach is described, which highlights an electrochemical reductive coupling as well as an intramolecular hydrogen atom transfer (HAT)-Giese addition to establish the 6,5-trans-carbon skeleton found in the Vitamin D family. Lastly, a late-stage decarboxylative cross-coupling approach allowed for the facile preparation of various C20-arylated derivatives which show promising biological activity in an early bioassay.
\end{abstract}

\section{[Introduction]}

Vitamin D (1, VitD) was identified in the early $20^{\text {th }}$ century as one of the essential molecules of the diet to support human life. A deficiency of $\mathrm{VitD}_{3}$ (4, Figure 1A) can result in a range of maladies such as a weakened immune system, rickets and osteomalacia. ${ }^{1}$ The structure of $\mathrm{VitD}_{3}$ was first elucidated by Windaus and Thiele in 1936 building on the findings of Askew et al. who demonstrated that $\mathrm{VitD}_{2}(\mathbf{5})$ could be derived from irradiation of ergosterol. ${ }^{2}$ Radiolabeling studies established the most active form of VitD in the body to be calcitriol (6). From 1965-1975, foundational pharmacological studies established the basic role that VitD plays in regulating serum calcium and phosphorus and bone homeostasis. Critically, VitD is also implicated in a number of biological processes specifically through the regulation of the transcription of hundreds of genes in a cell-specific fashion. As a consequence, starting in the 1950's, an enormous amount of effort was expended by medicinal chemists to search for VitD analogs that could exhibit prodifferentiating and antiproliferative effects on normal and cancer cells as well as immunomodulatory effects without causing hypercalcemia. ${ }^{1,3}$ Such programs resulted in the 
synthesis of $>3000$ modified VitD analogs and the commercial launch of about a dozen new medicines (e.g. Hectorol, Zemplar, Calcijex, Rocaltrol). ${ }^{3 \mathrm{c}, 4}$ Calcipotriol (7, Dovonex ${ }^{\mathrm{TM}}$, LEO Pharma) is currently the most successful VitD analog and is prescribed for the treatment of psoriasis, an autoimmune skin disease.

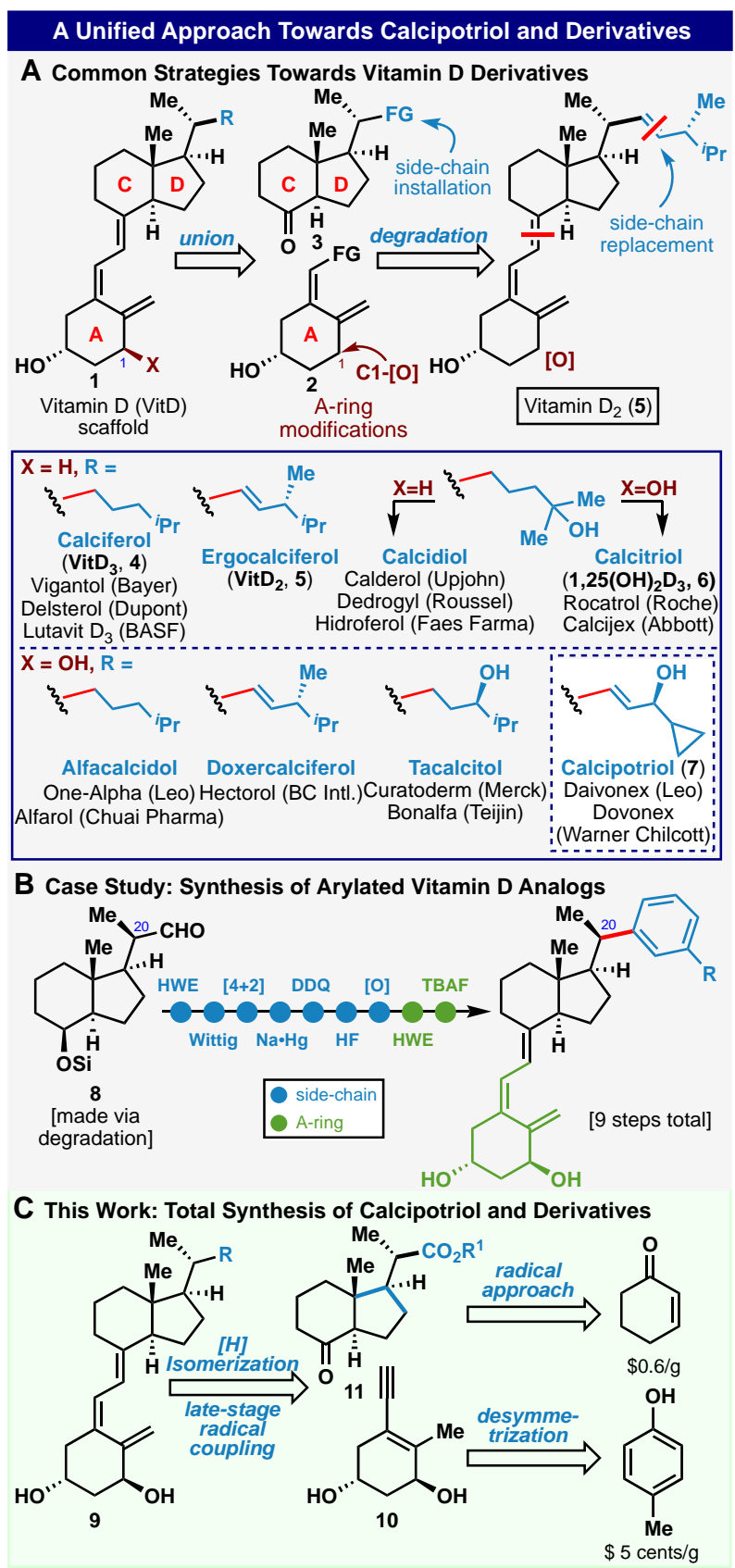

Figure 1. The rich history of the Vitamin $\mathrm{D}$ family. (A) Degradation of $\mathrm{VitD}_{2}$ still remains the premier approach in accessing novel VitD analogs on the side-chain. (B) Case study detailing the challenge of making new C20-arylated analogs. (C) Retrosynthetic analysis via a decarboxylative cross-coupling approach enables swift access to new analogs from inexpensive starting materials.

To date, the majority of approaches to access VitD derivatives (1) have utilized a convergent assembly of an A-ring fragment (2) with a fully formed CD bicycle (3, including the side-chain). 
As the side-chain appears to play a critical role in modulating bioactivity this results in very lengthy medicinal chemistry routes. ${ }^{5}$ The case study from LEO Pharma depicted in Figure 1B is emblematic of this challenge. Thus, in order to explore a simple aryl side-chain substituent, it must be tediously stitched onto the bicyclic aldehyde (8) [derived from degradation of $\mathrm{VitD}_{2}(\mathbf{5})$ ], followed by coupling to the A-ring thereby severely limiting accessible chemical space. The goal of this study was two-fold: (1) design an enantioselective, scalable, and convergent approach to 7 not wedded to semisynthesis; and (2) use of such a platform to create known and novel VitD analogs in a modular fashion (such as 1). Extensive explorations from both academic and industrial arenas in this area provided a useful foundation for this study and set the stage for a completely unique approach. In this Article, the realization of the plan outlined in Figure $1 \mathrm{C}$ through the strategic application of radical retrosynthesis ${ }^{6}$ for both ring annulation and substitution to address the $\mathrm{CD}$ rings, as well as symmetry recognition ${ }^{7}$ to scalably access ${ }^{8}$ ring $\mathrm{A}$ is described. This convergent approach enables late-stage side-chain installation onto triene $\mathbf{9}$ (a stable precursor to VitD analogs) prepared via the union of fully formed A-ring (10) and CD ring (11) fragments derived from inexpensive materials ( $p$-cresol and cyclohexanone, respectively).

\section{[Historical Context and Overall Retrosynthetic Plan]}

Just as the medicinal chemistry of steroids largely relies on a rich history of degradation and semisynthesis, explorations of the VitD class rely on such an approach. Thus, semi-synthesis has been employed by a variety of research programs, reported in ca. 90 publications and 90 patents to access thousands of VitD analogs (Figure $2 \mathrm{~A}) .{ }^{9}$ Specifically, VitD 2 could be oxidatively degraded to the so-called Inhoffen Lythgoe diol (12) containing the CD-ring system. Subsequent side-chain installation and reconstitution with a suitably functionalized A-ring surrogate (derived through synthesis) provided a multitude of VitD analogs and natural products (Path A, Figure 2A). In this way, many commercial medicines such as those outlined in Figure 1A have been discovered. An alternative approach (Path B, Figure 2A) often involves protection of the configurationally and chemically labile triene system (typically through cycloaddition with $\mathrm{SO}_{2}, 13$ ), followed by oxidative side-chain removal, reconstitution, and retro-cycloaddition/isomerization to unveil the bioactive VitD analog. Such a route is used by LEO pharma to manufacture calcipotriol (7), and requires a 14 step sequence including a tedious HPLC separation resulting from a lack of stereocontrol in a key reduction and several PG-manipulations. ${ }^{10}$

In contrast, the use of totally synthetic routes to access VitD analogs in an industrial setting, to the best of our knowledge, has been unreported. Such efforts have therefore been confined to the academic space where the VitD synthetic challenge has inspired numerous instructive pathways such as the five routes illustrated in Figure 2B. Path A represents an elegant example of how a cyclopropane (14) could be leveraged to protect the triene moiety yet it relies on diazo chemistry for its preparation which could be prohibitive on scale. ${ }^{11}$ Path B is the oldest strategy reported, employing a photochemical ring opening of 7-DHC derivatives (15) to allow for facile seco-Btriene formation. ${ }^{12}$ However, numerous redox manipulations to establish the triene core and requisite oxidation at $\mathrm{C} 1$ renders this approach less attractive. The most convergent approach that has received widespread adoption for the synthesis of the VitD core has been the Lythgoe-Roche strategy (Path C) between a trans-hydrindrane core 17 and advanced phosphonate 16. ${ }^{13}$ The synthesis of 16, initially developed by Baggiolini, was achieved in 13 steps, from (L)-carvone, and is even available commercially from a single vendor (Syncom BV). ${ }^{14}$ The coupling of this advanced substrate with 17 (Figure 2B) or a synthetically prepared trans-hydrindrane moiety (generally accessed through degradation of $\mathrm{VitD}_{2}$ ) provides the desired derivatives. 


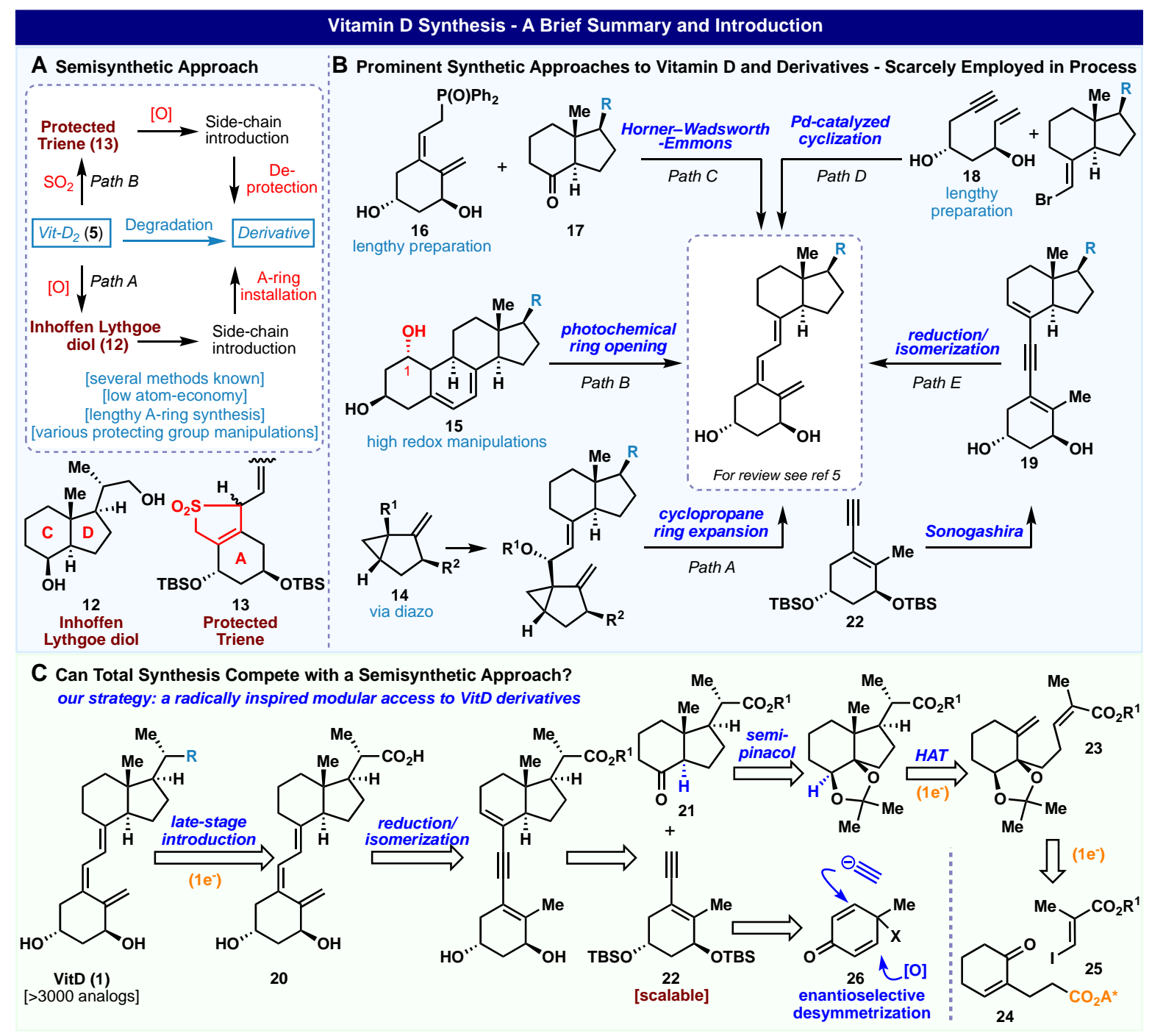

Figure 2. Current synthetic approaches towards the Vitamin D scaffold. (A) Semi-synthetic vs. protection/deprotection strategies. (B) Selected examples from various academic programs in accessing the VitD family. (C) Our retrosynthetic disconnections to access the triene system $\mathbf{1}$.

Another well- adopted strategy is the metal-catalyzed cycloisomerization approach, pioneered by Trost and co-workers as shown in Path D. ${ }^{15}$ This clever strategy employs a vinyl halide, typically prefunctionalized with the desired side-chain derivative, together with seco-A-ring synthon enyne 18. As such, this powerful method allows for the direct A-ring and triene formation in a single reaction step, however obtaining the acyclic enyne $\mathbf{1 8}$ with high enantiopurity has proven to be a challenge. ${ }^{5}$ Finally, Mourino and co-workers initially developed the reduction/isomerization approach to access the requisite triene system found in the Vitamin D scaffold (Path E). ${ }^{16}$ This approach relies on a semihydrogenation/thermal isomerization of dienyne $\mathbf{1 9}$ to the desired scaffold. Given the ease with which such an approach could potentially be performed on process scale, the convergency it enables, and the hidden symmetry of a building block such as $\mathbf{2 2}$, Path E directly influenced our initial disconnection.

The mission of the collaborative endeavor between the LEO and Scripps teams was to devise a patentable strategy that built off of the extensive literature precedent and in-house experience that 
would offer an alternative to semi-synthesis both for the commercial preparation of $\mathbf{7}$ and analogs thereof. Figure $2 \mathrm{C}$ outlines the blueprint that was devised to achieve this goal. Thus, VitD analogs (1) differing at the key side-chain could conceivably be accessed through a late-stage decarboxylative cross-coupling between triene $\mathbf{2 0}$ and a suitable coupling partner followed by reduction/isomerization. This modern disconnection could even be employed in a semi-synthetic approach to rapidly access new chemical space while the full total synthesis was developed. The dienyne precursor of $\mathbf{2 0}$ could be disconnected into fragments $\mathbf{2 1}$ and $\mathbf{2 2}$. Since the former fragment could be accessed easily using semisynthesis, it was critical that the route to the latter building block be robust and scalable. Nevertheless, a scalable route to $\mathbf{2 1}$ was devised based on the strategic combination of semi-pinacol and HAT annulation tactics from diene 23. This diene could then be accessed through a recently devised ${ }^{17}$ electrochemical reductive cross-coupling between enone 24 and vinyl iodide 25. For the A-ring fragment 22, an ambitious desymmetrization approach was targeted by engaging a suitably substituted dienone $\mathbf{2 6}$.

\section{[Scalable, Enantioselective Synthesis of the A-Ring]}

At the outset of this work, certain criteria needed to be met from the standpoint of starting material and reagent cost so as to be competitive with the semi-synthetic approach on scale. Aside from keeping the step count low, a fully stereocontrolled route was needed to avoid tedious separations on scale. Ideally, a process-friendly blueprint that would feature crystalline intermediates, no cryogenic reaction conditions, and inexpensive reagents was targeted. The classic strategy of cyclohexadienone desymmetrization ${ }^{18}$ was strategically appealing due to the low cost of the aromatic starting materials and the multitude of options for functional group installation. To be sure, enyne $\mathbf{2 2}$ could conceivably be accessed through the stepwise difunctionalization of a symmetrical pro-chiral dienone 23 (Figure 3A). As such, two parallel pathways leading to the same advanced intermediate 24 can be envisaged, which could be transformed to the final A-ring enyne 22 following diastereoselective ketone reduction and subsequent dehydration. Specifically, access to enantiopure $\mathbf{2 4}$ could potentially be achieved through either an enantioselective alkynylation or hydroxylation or a formal equivalent thereof. The ability to interchangeably employ either path with multiple tactics proved appealing from a route-scouting standpoint. For either route the initial desymmetrization requires both high regio- and facial selectivity to control the ee and de, respectively (see Figure $3 \mathrm{~A}$ ). The fact that the $\mathrm{C}-5 / \mathrm{C}-10$ stereocenters are eventually ablated after dehydration provided additional flexibility in the design. Such logic has been shown to be a powerful strategy to create multisubstituted cyclohexane scaffolds in a stereocontrolled fashion. ${ }^{18}$ Finally, since A-ring building block 22 shares the same formal oxidation state as benzene, all reactions need to be carefully orchestrated to avoid potential re-aromatization/decomposition.

Some initial forays to deconvolute this maze of options are outlined in Figure 3B. Path A, which involves an initial conjugate addition followed by formal b-oxygenation was evaluated first. In a racemic approach, initial conjugate addition worked smoothly followed by diastereoselective epoxidation and global reduction to open the epoxide and access the correct alcohol stereochemistry in $\mathbf{2 2}$. Unfortunately, this concise route could not be rendered enantioselective despite extensive efforts (see SI for a summary). The route was nevertheless instructive in that it set an important precedent for the diastereoselective installation of the critical C-1/C-3 hydroxyl groups in 22. Concurrent with this approach, an ambitious proposal to enlist the Zard-alkyne synthesis was pursued wherein an isoxazolone serves as a surrogate for the unveiling of an alkyne. ${ }^{19}$ Although isoxazolone engaged in a Michael addition to deliver 25, such an adduct could 
A Strategies Towards the Synthesis of A-Ring Synthon 22

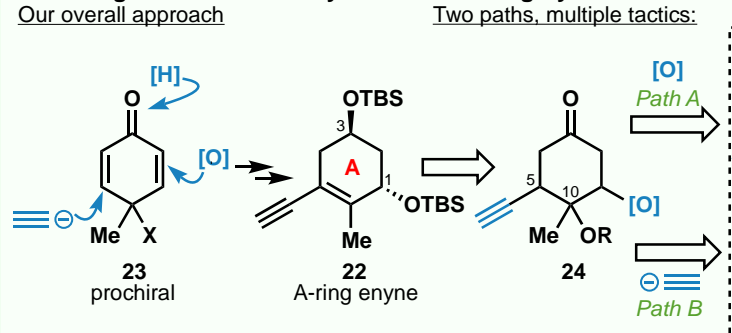<smiles>CCC1(O)C=CC(=O)C=C1</smiles>

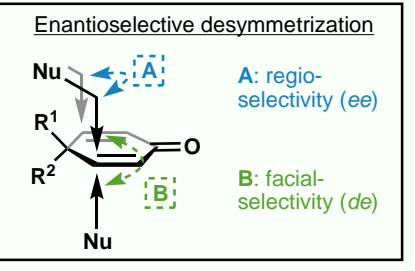

B Initial Forays Towards Enyne 22
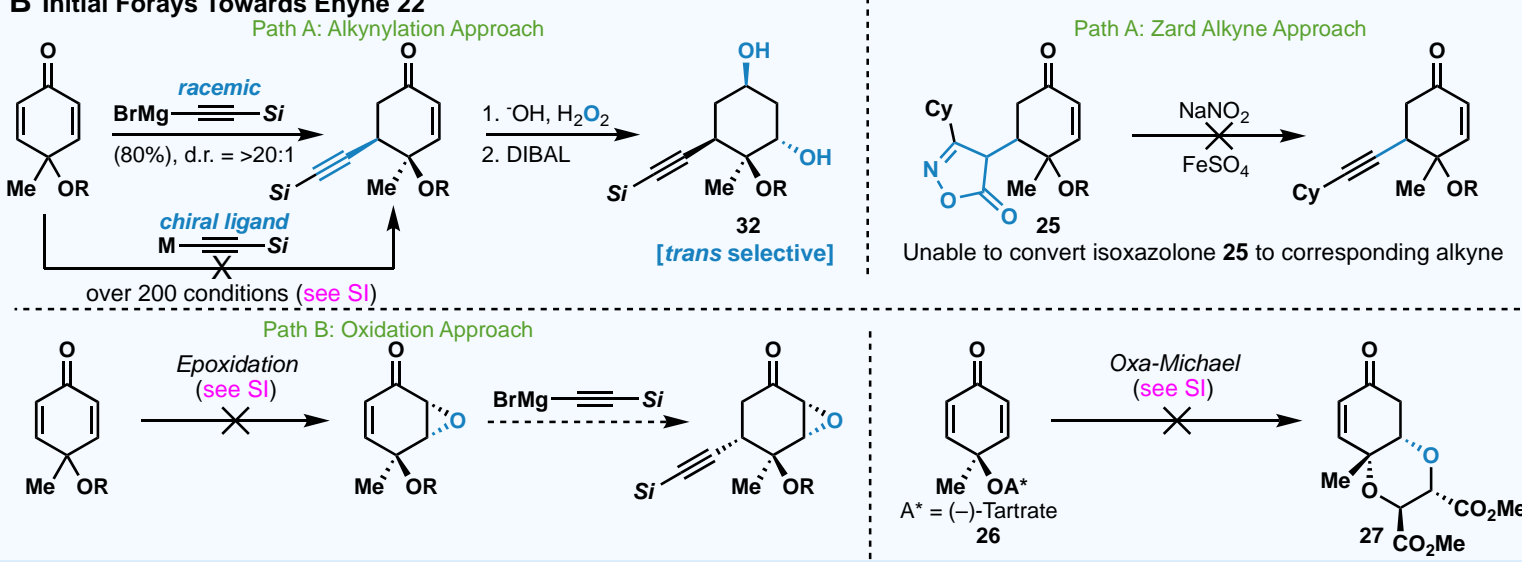

C Enantioselective Borylation Enables the Synthesis of A-Ring Precursor 22

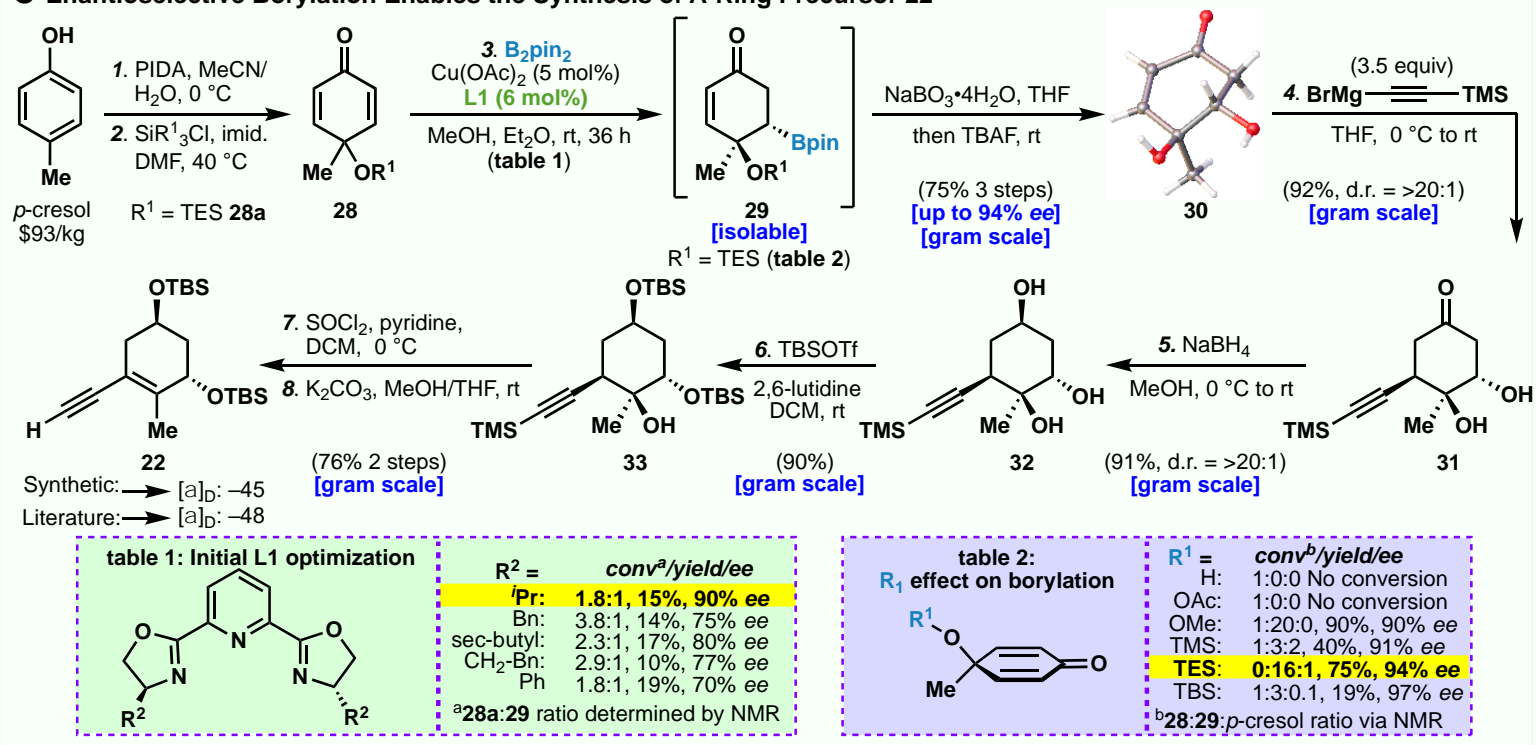

Figure 3. A new approach towards the synthesis of A-ring precursor 22. (A) Synthetic planning and potential pitfalls. (B) Early-stage development and key lessons learned. (C) Final synthetic route towards enantiopure A-ring precursor 22.

not be unraveled to provide the requisite alkyne. Turning to Path $\mathrm{B}$, the most intuitive approach of enantioselective epoxidation was surveyed. However, as with the aborted route described above, the racemic pathway delivered the desired diastereomeric diol $\mathbf{2 4}$ but could not be rendered enantioselective in a practical fashion. ${ }^{20}$ Finally, an approach inspired by You's work on 
desymmetrization of cyclohexadienones via Brønsted acid-catalyzed enantioselective oxaMichael reaction was pursued by targeting tartrate-derived dienone $26 .{ }^{21}$ The tartrate was expected to act as an auxiliary to introduce the chirality, however, this target proved to be inaccessible in synthetically useful yields (3-5\% observed after screening multiple conditions for oxidative dearomatization). It is postulated that $p$-cresol oligomerization was faster than the nucleophilic attack resulting in the low yield of 27.

We next investigated alternate oxidation approaches, in particular borylation-oxidation. Although the desymmetrization of 1,4-dienones via borylation is not known, there are examples of asymmetric borylation on both acyclic and cyclic enones. ${ }^{22}$ As mentioned previously, while the dienone substrate appeared to be unreactive under most epoxidation conditions, borylation of dienone 28a under Kobayashi's borylation conditions gave the desired product in racemic form. ${ }^{22 \mathrm{~g}}$ Translation of these conditions to the asymmetric version proved challenging wherein Kobayashi's bipy-derived ligand failed to deliver any appreciable yields of the desired borylated compound 29; however, a survey of various BOX ligands led to the discovery that the commercially available $(R, R)-{ }^{i}$ Pr-Pybox $\mathbf{L} 1$ readily desymmetrized the molecule in excellent enantioselectivity albeit in low yields. Furthermore, there seemed to be a delicate balance between conversion and enantioselectivity at the 4-hydroxy position, wherein the judicious choice of a TES protecting group proved optimal for both ee and overall yield (Figure 3C, table 2). After further optimization, this symmetry-breaking borylation-oxidation sequence could be performed on $50 \mathrm{mmol}$ scale with $75 \%$ yield and $94 \%$ ee over 3 steps (see SI for full details). Importantly, the use of L1 in process chemistry is precedented. ${ }^{23}$

With enantiopure diol 30 in hand, attention turned towards installing the requisite alkyne and internal olefin found in enyne 22. Conjugate addition of the resulting dihydroxyenone $\mathbf{3 0}$ occurred with complete diastereoselective control to deliver ketone $\mathbf{3 1}$ in excellent yield. It is worth noting that the chelation-controlled alkynylation proved highly chemoselective even at ambient temperature. $\mathrm{NaBH}_{4}$ reduction of 31 proceeded with high trans selectivity (d.r. $=>20: 1$, relative stereochemistry confirmed via $\mathrm{nOE}$ ), followed by selective bis-TBS protection of triol $\mathbf{3 2}$ to deliver tertiary alcohol 33. It was anticipated the elimination of alcohol $\mathbf{3 3}$ would prove challenging due to its inaccessibility, however after various attempts (see the SI), the use of $\mathrm{SOCl}_{2}$ in pyridine/DCM smoothly provided the desired enyne 22 after TMS deprotection.

To summarize the route to enyne $\mathbf{2 2}$, a highly enantioselective conjugate borylation of an easily accessible cyclohexadienone sets the stage for all subsequent transformations to occur with both high chemo- and diastereoselective control. From an efficiency and scalability perspective, it traverses through 3 isolated intermediates from inexpensive $p$-cresol and can be conducted at noncryogenic temperatures on multi-gram scale. This route thus represents one of the most processfriendly paths to date to a fully synthetic A-ring VitD module.

\section{[The CD-Ring Fragment: Past Strategies, Challenges, and the Development of a New Approach]}

Within the rich history of steroid synthesis, one extremely popular chapter involves approaches to the CD-ring system, a deceptively simple looking fused bicycle (Figure 4). This thermodynamically unfavored trans-hydrindane (6,5-trans-fused) core is inherently strained, rendering syntheses of this bicycle challenging. The most popular general strategies are summarized in Figure 4B. Amongst all the total synthetic efforts towards the CD ring fragment $(>100$ !), few examples can deliver this structure within ten steps. 


\section{CD-Ring Synthesis - an Ongoing Synthetic Challenge in Steroid Chemistry}

A Synthetic Considerations

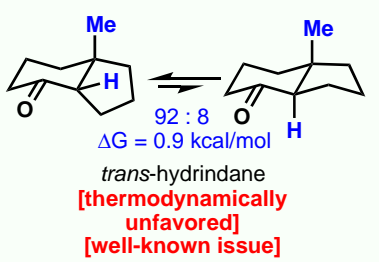

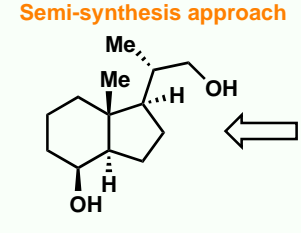

Inhoffen-Lythgoe diol

B Prominent Synthetic Approaches to CD-ring

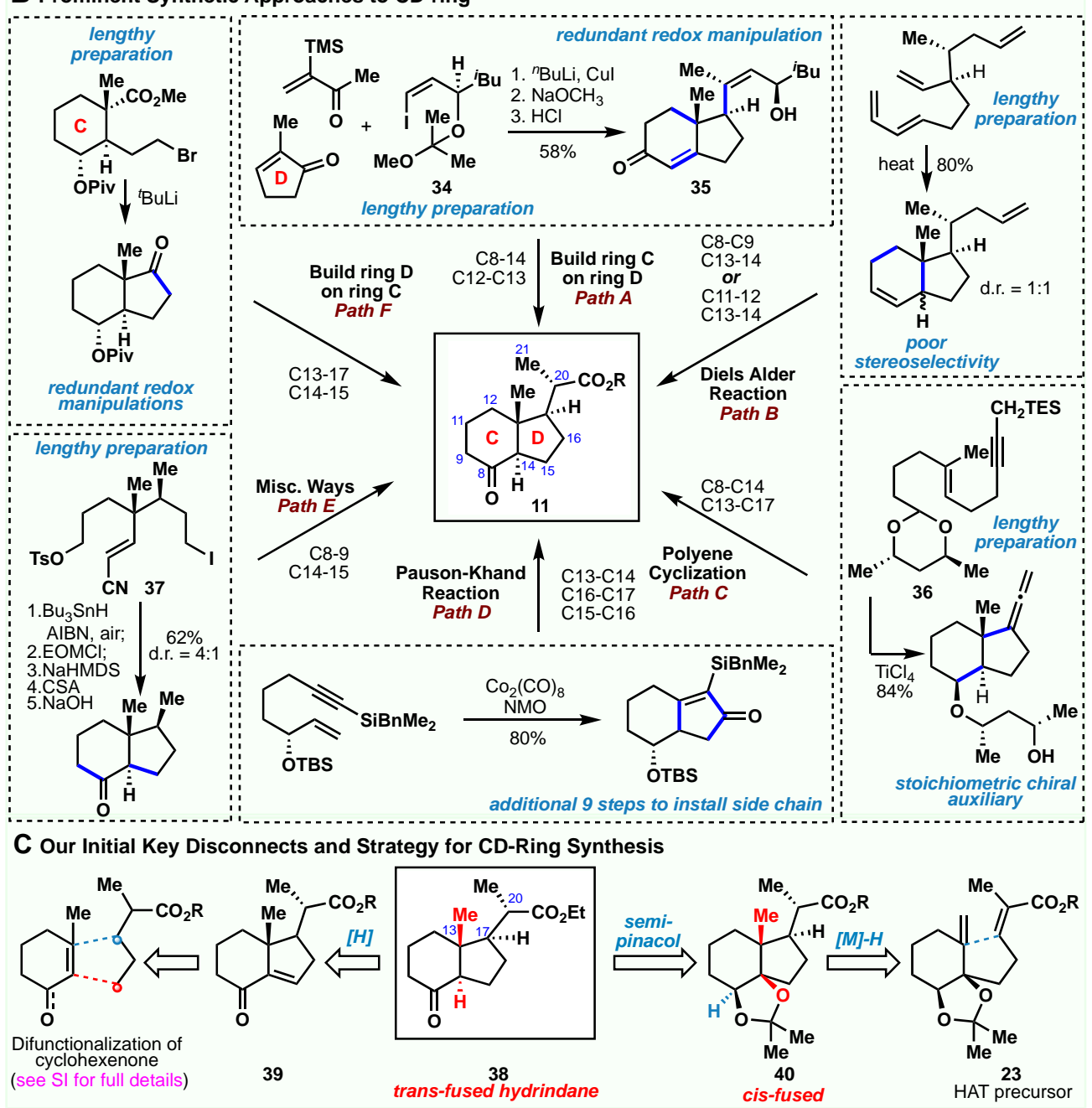

Figure 4. Past and current approaches towards the 6,5-trans-fused skeleton found in steroid chemistry and current approach relying on a semi-pinacol rearrangement.

In the vast majority of cases, the starting materials for the key ring constructing step either required lengthy routes or extensive concession steps ${ }^{24}$ (redundant redox manipulations and functional group interconversions) were employed after the ring system had been constructed. For the specific purpose of synthesizing VitD, key intermediate 11 with oxygenation at C-8 was targeted. Many of the routes that are used to access a CD-ring precursor for steroids are not easily adapted for VitD. For example, the elegant 3-component coupling between 2-methylcyclopentenone, a vinylsilyl methyl ketone, and an optically active iodo-olefin 34 (4-step synthesis from leucine) reported by 
Tsuji and co-workers arrived at $\mathbf{3 5}$ and was shown by others to require additional steps to forge the trans-hydrindrane (Path A). ${ }^{25}$ Wilson's intramolecular Diels-Alder approach to a minimally functionalized ring system required a lengthy sequence (racemic) to the unsaturated precursor and resulted in poor diastereocontrol (Path B). ${ }^{26}$ Johnson's classic cation-olefin cyclization approach (Path C) successfully forged the trans-6-5 ring system (87:13 d.r., 82\% isolated yield) however precursor 36 was arduous to prepare ( 8 steps and an expensive non-recyclable chiral auxiliary) and the resulting allene required several additional steps to install the side-chain functionality. ${ }^{27}$ The Mourino group utilized a Pauson-Khand cyclization (Path D) to form the CD-ring skeleton and a Si-assisted allylic substitution to set the challenging pivotal quaternary methyl group at the fusedring junction of the CD-trans-hydrindane core followed by an additional 9 steps to install the side chain $^{28}$. Takahashi reported a radical cyclization of 37 (10-step preparation), provided the transring fusion after anionic ring cyclization (Path E). ${ }^{29}$ The C-ring could also be installed first followed by cyclization of ring-D as exemplified by Stork's classic approach (Path F). ${ }^{30}$ As with the other routes, this indirect path required multiple steps. As mentioned above, the six examples shown here are not a comprehensive summary, but rather a selection of strategically diverse synthetic blueprints to this ring system known at the outset of these studies. Ultimately, the HajosParrish ketone (13) is historically one of the most popular starting materials for total synthesis approaches when the $\mathrm{CD}$ ring system is retained.

By far, the most practical approach to $C D$ fragments remains semi-synthesis via vitamin $\mathrm{D}_{2}$ degradation (to the Lythgoe-Inhoffen diol, 12). ${ }^{3}$ Indeed, LEO Pharma employed this starting material for their in-house medicinal chemistry efforts (Figure 1B). ${ }^{31}$

In contemplating a new approach to this strained 6,5-fused bicyclic system in 38, two strategies emerged to the forefront (Figure 4C). Late-stage stereocontrolled hydrogenation of olefin 39 or semi-pinacol rearrangement of protected diol 40. For the former approach, numerous concise routes were designed (see SI for examples). In order to de-risk the path, 39 could be prepared from VitD2 semisynthetically. Unfortunately, despite screening dozens of reduction conditions, the coveted trans-stereochemistry could never be obtained. Efforts then shifted completely to the latter semi-pinacol approach. The advantage of this design was that the trans-stereochemistry could be pre-programmed by virtue of the diol precursor 40. In turn, this isopropylidene-protected diol served as a rigid scaffold (cis-6,5 bicycle) that could potentially control the stereochemical outcome of an intramolecular reductive HAT-based olefin coupling ${ }^{32}$ on substrate 23. Such a disconnection was ambitious since it would need to controllably form a key quaternary center and two contiguous tertiary stereocenters in one step. From modeling studies there was confidence that the key C13-C17 bond would form in the desired fashion, although the stereochemical outcome at C20 was uncertain. HAT-precursor $\mathbf{2 3}$ could be traced back to inexpensive 2-cyclohexenone and an acrylate.

Our initial entry towards the enantioselective synthesis of advanced CD-ring $\mathbf{3 8}$ commenced by targeting enoate 40 (Figure 5). Baylis-Hillman reaction on 2-cyclohexenone with $t$-butyl acrylate in the presence of catalytic DBU delivered enone $\mathbf{4 1}$ in $81 \%$ yield on multi-gram scale. ${ }^{33}$ Although various disconnections and conditions were employed to deliver the diol in $\mathbf{4 2}$ (see SI), an asymmetric dihydroxylation of such an olefin would appear the most appealing choice. Although using NMO as the co-oxidant and citric acid in the presence of catalytic OsO4 efficiently delivered the dihydroxy product in a racemic fashion, ${ }^{34}$ the asymmetric version proved more challenging. Indeed, dihydroxylation on electron deficient alkenes suffers from poor enantioselectivity, especially in endocyclic systems. ${ }^{35}$ Moreover, alkyl $\alpha$-substitutions further exacerbate this issue, 


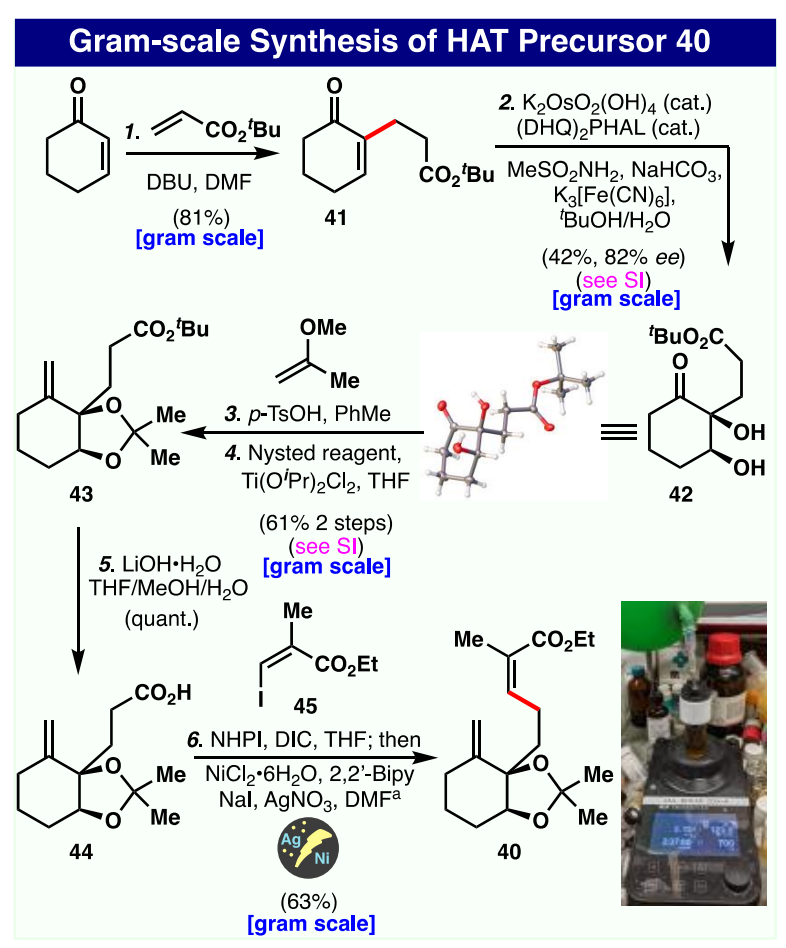

Figure 5. Application of the Ag-nanoparticle functionalized electrochemical reductive coupling enables gram-scale access to enoate 40. ${ }^{a}$ Electrochemical conditions: (+)Mg/ (-)RVC 6 mA, $3.3 \mathrm{~F} / \mathrm{mol}$.

and is known to interfere with the coordination between the osmium catalyst and alkene. Supported by the fact that there are only a few examples on asymmetric dihydroxylation of cyclopentenone or cyclohexenone with $\alpha$-substitutions, ${ }^{36}$ dihydroxylation on such systems still prove challenging today.

In the initial optimization, potassium ferrocyanide(III) was able to achieve the desired dihydroxy ketone 42 in $66 \%$ ee. Consistent with Sharpless's original study on dihydroxylation of $\alpha, \beta-$ unsaturated ketones, ${ }^{35 \mathrm{~b}}$ the reaction utilizing $\mathrm{AD}$-mix only gave trace amount of $\mathbf{4 2}$, requiring extra osmium due to the less reactive olefin as well as additional base to buffer the reaction. After screening a variety of additives, ligands and various solvent combinations (see SI), diol $\mathbf{4 2}$ was obtained with $67 \%$ conversion and $74 \%$ ee. In its most optimized form, diol $\mathbf{4 2}$ could be obtained in $42 \%$ yield with $82 \%$ ee on gram scale. The desired enantiomer could be enantioenriched through recrystallization (gram scale).

With ample quantities of diol $\mathbf{4 2}$ in hand, protection with 2-methoxypropene gave the corresponding acetonide which served to rigidify the system. Subsequent methylenation required an extensive optimization as standard olefination conditions (Wittig, salt-free Wittig, Peterson olefination) gave low conversion or low yields on scale, while most Ti-based reagents (TakaiUtimoto, Lambardo, Petasis) proved similarly unsuccessful. The use of the Ti-based Nysted reagent ultimately delivered olefin $\mathbf{4 3}$ in respectable yields. ${ }^{37}$ After further optimization (see SI), the use of Nysted reagent, in conjunction with $\operatorname{Ti}\left(\mathrm{O}^{i} \mathrm{Pr}\right)_{2} \mathrm{Cl}_{2}$, delivered the desired olefin 43 in $61 \%$ (2 steps from diol 42, gram-scale). ${ }^{38}$

Saponification of tert-butyl ester $\mathbf{4 3}$ delivered acid $\mathbf{4 4}$ in quantitative yield, which then set the stage for a key $\mathrm{C}-\mathrm{C}$ bond forming step (44 to $\mathbf{4 0}$ ). A number of approaches were evaluated for achieving this conversion on $\mathbf{4 4}$ and related intermediates; ultimately, the newly developed electrochemical 
reductive cross-coupling ${ }^{17}$ proved to be successful in accessing advanced intermediate $\mathbf{4 0}$. Thus, in-situ activation of acid $\mathbf{4 4}$ with NHPI, followed by reductive coupling with vinyl iodide $\mathbf{4 5}$, enabled by Ag-nanoparticle functionalized electrochemical cross-coupling ${ }^{17}$, delivered HAT precursor 40 in $48 \%$ yield on small-scale. Gratifyingly, a correlation of yield and reaction concentration was observed, providing $\mathbf{4 0}$ in 63\% yield (gram-scale).

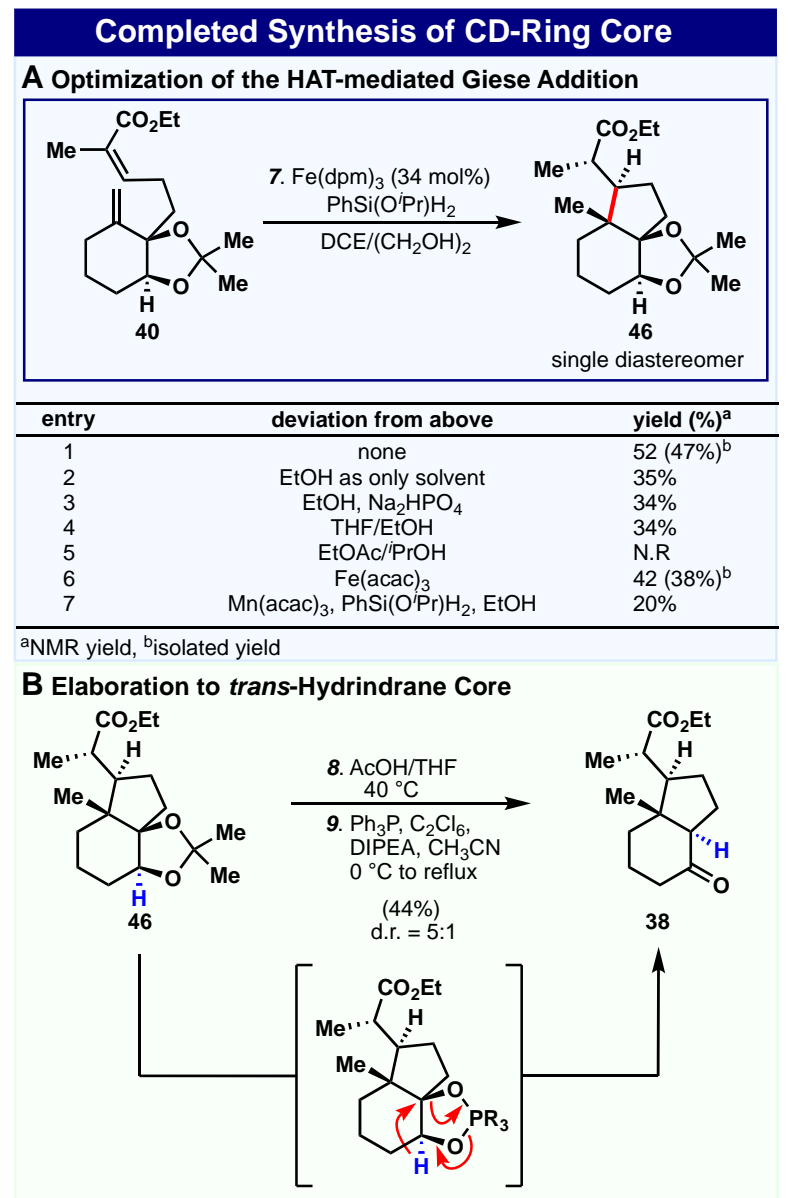

Figure 6. Completion of the trans-fused 6,5-ring system found in VitD.

With enoate 40 in hand, attention turned towards establishing the CD-ring core via an intramolecular HAT-mediated annulation (Figure 6A). ${ }^{32}$ During the course of extensive optimization (see SI for full details), the utilization of $\mathrm{PhSi}\left(\mathrm{O}^{i} \mathrm{Pr}\right) \mathrm{H}_{2}$ was found to be crucial, whereas phenyl silane failed to give 46. In general, the solvent choice had very little effect on the reaction conversion (entries 1-4), although ethyl acetate/ ${ }^{i} \mathrm{PrOH}$ proved detrimental (entry 5). A survey of catalysts revealed that manganese(III)-based catalysts only led to unsatisfactory yields (entry 7), while the use of Fe-based catalysts $\left(\mathrm{Fe}(\mathrm{dpm})_{3}, \mathrm{Fe}(\mathrm{acac})_{3}\right)$ delivered the desired annulated product in appreciable yields (entries 1,6). After a thorough screening of conditions, the use of $\mathrm{Fe}(\mathrm{dpm})_{3}$ and $\mathrm{PhSi}\left(\mathrm{O}^{i} \mathrm{Pr}\right) \mathrm{H}_{2}$, in $\mathrm{DCE} /(\mathrm{CH} 2 \mathrm{OH})_{2}$ delivered the desired stereoisomer 46 in $54 \%$ isolated yield. It is noteworthy that this HAT-mediated cyclization installs 3 contiguous stereocenters with the major diastereomer being the correct C20 stereochemistry belonging to the VitD family. 
Having established the VitD core, the pivotal late-stage semi-pinacol rearrangement of protected diol $\mathbf{4 6}$ towards ketone $\mathbf{3 8}$ was explored. In the event, the key diol was liberated upon treatment with acetic acid at $40{ }^{\circ} \mathrm{C}$. Treatment of the crude diol after workup with $\mathrm{Ph}_{3} \mathrm{PCl}_{2}$ induced a semipinacol rearrangement to deliver ketone $\mathbf{3 8}$ in $44 \%$ yield (over 2 steps), presumably via a phosphorane intermediate (see Figure 6B), to complete the asymmetric synthesis of the CD-ring fragment. $^{39}$

\section{[Late-Stage Side Chain Installation: Synthesis of Calcipotriol and analogs thereof]}

With scalable access to both A-ring precursor $\mathbf{2 2}$ and keto-ester $\mathbf{3 8}$, the union of both intermediates was pursued to complete the total synthesis of 7 and provide access to various side-chain derivatives. To this end, keto-ester 38 was converted to vinyl triflate 47, followed by Sonogashira coupling with A-ring subunit 22 via conditions established by Mourino and co-workers to deliver en-yn-ene 48 in $71 \%$ yield over 2 steps. ${ }^{16 c, 40}$ Saponification of ethyl ester $\mathbf{4 8}$ followed by conversion to corresponding NHPI ester proceeded smoothly in delivering advanced redox-active (RAE) intermediate 49 in $60 \%$ yield over 2 steps.

Numerous conditions were evaluated to couple vinyl halide precursor 50 (5-steps from commercially available cyclopropanecarbonyl chloride, see SI). After exhaustive screening of various RAEs and suitable cross-coupling partners $(\mathbf{5 0}, \mathrm{X}=\mathrm{I}, \mathrm{Br}, \mathrm{ZnR})$ based on previous reported conditions, ${ }^{32 b, 41}$ the direct coupling could be practically achieved only under newly established electrochemical conditions, with vinylbromide 50A being the most suitable choice for this transformation (see SI). ${ }^{17}$ In the event, RAE 49 and 50A were subjected to Ag-nanoparticle based electrochemical conditions in delivering $\mathbf{5 1}$ in $48 \%$ NMR yield (carried crude to the next step) as a 1:1 mixture of diastereomers (ca. $75 \mathrm{mg}$ scale). The endgame sequence involved tandem semihydrogenation/isomerization followed by global deprotection to furnish calcipotriol in $38 \%$ isolated yield over 3 steps from 49.

To facilitate access to arylated VitD analogs that had previously required ca. 9 steps to prepare (Figure 1B), late-stage side-chain installation of triene $\mathbf{5 2}$ was pursued (9, Figure 1C). Thus, enyn-ene $\mathbf{4 8}$ was transformed to triene acid $\mathbf{5 2}$ via a two-step protocol (reduction/isomerization, saponification) in $46 \%$ isolated yield (over 2 steps). Fe-catalyzed decarboxylative arylation, ${ }^{41 \mathrm{f}}$ via in-situ activation of acid $\mathbf{5 2}$ with HATU and addition of the corresponding Ar2Zn in the presence of $20 \mathrm{~mol} \% \mathrm{Fe}(\mathrm{acac}) 3$, provided the desired arylated VitD analogs in appreciable yields (53-57, $21-40 \%)$ as a mixture of diastereomers. The value of this approach is evident by the synthetic short-cut that can be taken to access such arylated calcipotriol analogs. Convergent access to such analogs is facilitated by the chemoselective nature of the decarboxylative coupling that does not require protection of the reductively, oxidatively, and photochemically sensitive triene system that historically requires an additional round of protection. 
Synthesis of Calcipotriol and Medicinally Relevant C20-Analogs

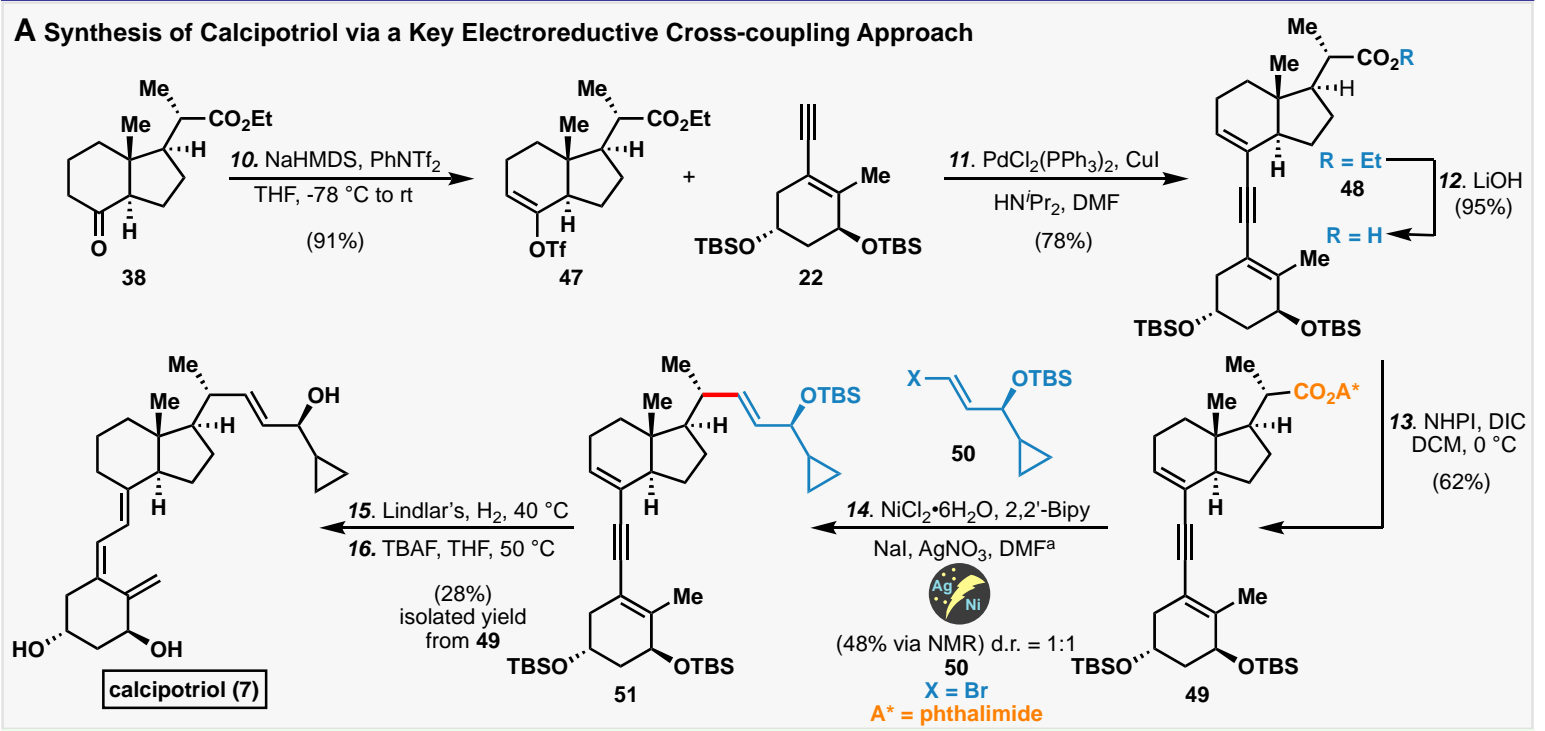

B Late-stage Access to Medicinally Relevant C20-Analogs via 1e- Approach

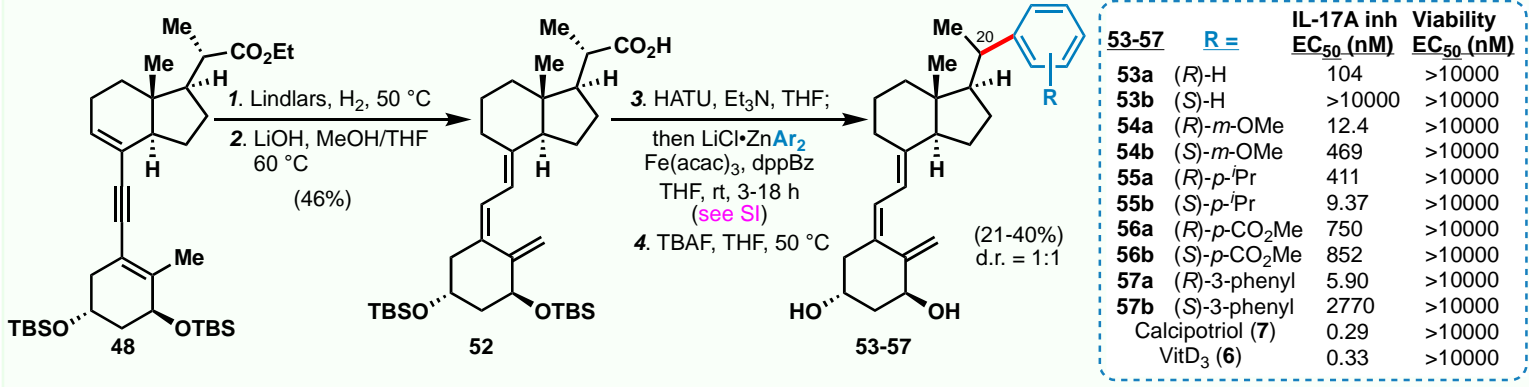

Figure 7. Total synthesis of Calcipotriol (7) and late-stage application of the Fe-catalyzed decarboxylative crosscoupling allows for the rapid access of C20-arylated analogs which display promising EC50 values in an early

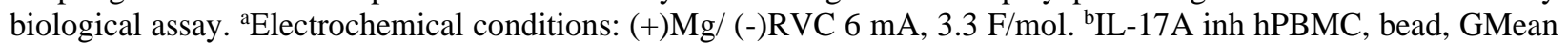
Rel EC50 (nM). ${ }^{c}$ Viability hPBMC bead, GMean Rel EC50 (nM).

\section{[Bioactivity Evaluation of C20-Arylated Vit D Analogs]}

With C20-analogs 53-57 in hand, attention turned towards determining their cellular efficacy and overall potency in a human PBMCs assay measuring the secretion of IL-17A. IL-17A is a major effector cytokine in the pathogenesis of psoriasis, and antibodies targeting IL-17A or its receptors have shown to be highly efficacious in psoriasis patients (e.g. calcipotriol (7) potently inhibits the secretion of IL-17A). ${ }^{42}$ In the assay event, human PBMCs were supplemented with IL-23 to stimulate the Th17 pathway and incubated with T-cell receptor crosslinking antibodies antiCD3/antiCD28 for 3 days to stimulate the secretion of IL-17A.

Comparison of the activity of C20-arylated vitamin D analogs 53-57 in the human PBMC IL-17 release assay identified several potent compounds, although a moderate overall loss in potency was observed compared to calcipotriol (7) (Figure 7). In general, a clear difference in potency was observed between the two C20-stereoisomers of each arylated compound tested. Hence, $(R)$-53a showed an $\mathrm{EC}_{50}$ of $104 \mathrm{nM}$ while the corresponding $(S)$-53b exhibited a complete loss of activity. $(R)-54 a$, bearing a -OMe group, resulted in a 9-fold increase in potency compared to 53a, while its $(S)$-isomer counterpart in 54b showed only moderate activity. Interestingly in the case of the 
para-substituted ${ }^{i} \operatorname{Pr}$ analogs $\mathbf{5 5 a}$ and $\mathbf{5 5 b}$, an inverse in potency between the two isomers of 55 was observed wherein the $(S)$-isomer 55b was approximately 40 -fold more potent than the $(R)$ isomer 55a. An overall marked loss in activity was seen for both isomers of the $p-\mathrm{CO}_{2} \mathrm{Me}$ analogs in 56. The meta-biphenyl compound 57a was the most potent of the analogs tested with an $\mathrm{EC}_{50}$ of $5.9 \mathrm{nM}$, while a near-complete loss of activity was observed for its corresponding isomer $\mathbf{5 7 b}$. Gratifyingly, none of the compounds tested showed any toxicity $\left(\mathrm{EC}_{50}>10 \mu \mathrm{M}\right)$ in a human PBMC viability assay.

\section{[Conclusions]}

In conclusion, a completely synthetic approach, not wedded to semi-synthesis, towards the scalable synthesis of calcipotriol and related analogs is described. Several steps are worth noting: 1) symmetry recognition to scalably access the A-ring via an unprecedented enantiocontrolled conjugate borylation of a cyclohexadienone; 2) strategic application of radical retrosynthesis for the rapid development of the $\mathrm{CD}$ ring using recently developed methods such as Ag-nanoparticle enabled electrochemical reductive cross coupling and a highly diastereoselective HAT-mediated annulation; 3) implementation of a semi-pinacol rearrangement to address the thermodynamically unfavored 6,5-trans ring fusion; 4) modular access to medicinally relevant analogs bypassing the need for a custom route for each derivative that is again reliant on Ag-nanoparticle electrochemical reductive cross coupling; and 5) scalability of routes to key fragments 47 and 22 via inexpensive starting materials without the need of any cryogenic temperatures.

This modern take on the synthesis of a classic molecule (Vitamin D) builds on the rich history of prior syntheses and provides uniquely efficient access to both natural and unnatural analogs for exploration in medicine.

\section{ASSOCIATED CONTENT}

\section{Supporting Information}

Experimental procedures, characterizations, and the detail of DFT calculation.

\section{AUTHOR INFORMATION}

Corresponding Author

*pbaran@scripps.edu

\section{Author Contributions}

The manuscript was written through contributions of all authors. All authors have given approval to the final version of the manuscript.

\section{Notes}

${ }^{\S}$ J.G. and K.X.R. contributed equally to this work. L. Jørgensen and T. Skak-Nielsen are employees of LEO Pharma A/S.

\section{ACKNOWLEDGMENT}


Financial support for this work was provided by LEO Pharma; K.X.R was supported by NIH (PA18-586) and M.O. was supported by the Polish National Agency for Academic Exchanged (the Bekker programme no. PPN/BEK/2020/1/00111/DEC/1). We are grateful to Dr. Dee-Hua Huang and Dr. Laura Pasternack (Scripps Research) for assistance with nuclear magnetic resonance (NMR) spectroscopy; M. Gembicky (University of California, San Diego) for assistance with Xray crystallography; J. S. Chen, B. B. Sanchez, and E. Sturgell (Scripps Research) for assistance with LCMS analysis and HRMS analysis; and Birte Thoke-Jensen (LEO Pharma) for assistance on the PBMC IL-17A assay.

\section{REFERENCES}

1. Bikle, D. D. Chem Biol 2014, 21, 319.

2. (a) Windaus, A.; Schenck, F.; Werder, F. V. Z. Physiol. Chem. 1936, 241, 100. (b) Askew, F. A.; Bruce, H. M.; Callow, R. K.; Philpot, J. S.; Webster, T. A. Nature (London) 1931, 128.

3. (a) Autier, P.; Boniol, M.; Pizot, C.; Mullie, P. The Lancet Diabetes \& Endocrinology 2014, 2, 76. (b) Feldman, D.; Krishnan, A. V.; Swami, S.; Giovannucci, E.; Feldman, B. J. Nature Reviews Cancer 2014, 14, 342. (c) Jones, G. Endocrinology and Metabolism Clinics of North America 2010, 39, 447. (d) Leyssens, C.; Verlinden, L.; Verstuyf, A. Front Physiol 2014, 5, 122.

4. Maestro, M. A.; Molnár, F.; Carlberg, C. J. Med. Chem. 2019, 62, 6854.

5. Zhu, G.-D.; Okamura, W. H. Chem. Rev. 1995, 95, 1877.

6. (a) Hung, K.; Hu, X.; Maimone, T. J. Natural Product Reports 2018, 35, 174. (b) Romero, K. J.; Galliher, M. S.; Pratt, D. A.; Stephenson, C. R. J. Chem. Soc. Rev. 2018, 47, 7851. (c) Smith, J. M.; Harwood, S. J.; Baran, P. S. Acc. Chem. Res. 2018, 51, 1807.

7. $\quad$ Bai, W.-J.; Wang, X. Natural Product Reports 2017, 34, 1345.

8. Kuttruff, C. A.; Eastgate, M. D.; Baran, P. S. Natural Product Reports 2014, 31, 419.

9. Number of publications and patents were accumulated by performing a scifinder reaction search of universal VitD analog skeleton.

10. (a) Calverley, M. J. Tetrahedron 1987, 43, 4609. (b) Sabroe, T. P.; Calverley, M. J. Stereoselective synthesis of vitamin D analogues.

11. (a) Wilson, S. R.; Serajul Haque, M.; Venkatesan, A. M.; Zucker, P. A. Tetrahedron Lett. 1984, 25, 3151. (b) Wilson, S. R.; Venkatesan, A. M.; Augelli-Szafran, C. E.; Yasmin, A. Tetrahedron Lett. 1991, 32, 2339.

12. (a) Barton, D. H. R.; Hesse, R. H.; Pechet, M. M.; Rizzardo, E. J. Am. Chem. Soc. 1973, 95, 2748. (b) Barton, D. H. R.; Hesse, R. H.; Pechet, M. M.; Rizzardo, E. J. Chem. Soc., Chem. Commun. 1974, 203. (c) Dauben, W. G.; Phillips, R. B. J. Am. Chem. Soc. 1982, 104, 355.

13. (a) Lythgoe, B.; Moran, T. A.; Nambudiry, M. E. N.; Ruston, S.; Tideswell, J.; Wright, P. W. Tetrahedron Lett. 1975, 16, 3863. (b) Posner, G. H.; Eydoux, F.; Lee, J. K.; Bull, D. S. Tetrahedron Lett. 1994, 35, 7541.

14. Baggiolini, E. G.; Iacobelli, J. A.; Hennessy, B. M.; Batcho, A. D.; Sereno, J. F.; Uskokovic, M. R. J. Org. Chem. 1986, 51, 3098.

15. (a) Trost, B. M.; Dumas, J.; Villa, M. J. Am. Chem. Soc. 1992, 114, 9836. (b) Trost, B. M.; Hanson, P. R. Tetrahedron Lett. 1994, 35, 8119. 
16. (a) Castedo, L.; Mascareñas, J. L.; Mouriño, A.; Sarandeses, L. A. Tetrahedron Lett. 1988, 29, 1203. (b) Castedo, L.; Mouriño, A.; Sarandeses, L. A. Tetrahedron Lett. 1986, 27, 1523. (c) Mascareñas, J. L.; Sarandeses, L. A.; Castedo, L.; Mouriño, A. Tetrahedron 1991, 47, 3485.

17. Harwood, S.; Palkowitz, M.; Gannett, C.; Perez, P.; Yao, Z.; Sun, L.; Abruña, H.; Anderson, S.; Baran, P. ChemRxiv. Cambridge: Cambridge Open Engage 2021, doi: 10.33774/chemrxiv.

18. Kalstabakken, K. A.; Harned, A. M. Tetrahedron 2014, 70, 9571.

19. (a) Boivin, J.; Elkaim, L.; Ferro, P. G.; Zard, S. Z. Tetrahedron Lett. 1991, 32, 5321. (b) Boivin, J.; Huppé, S.; Zard, S. Z. Tetrahedron Lett. 1995, 36, 5737.

20. (a) A. Clark, D.; de Riccardis, F.; C. Nicolaou, K. Tetrahedron 1994, 50, 11391. (b) Adam, W.; Lupón, P. Chem. Ber. 1988, 121, 21. (c) Corey, E. J.; Wu, L. I. J. Am. Chem. Soc. 1993, 115, 9327. (d) Li, C.; Johnson, R. P.; Porco, J. A. J. Am. Chem. Soc. 2003, 125, 5095.

21. (a) Gu, Q.; Rong, Z.-Q.; Zheng, C.; You, S.-L. J. Am. Chem. Soc. 2010, 132, 4056. (b) Rubush, D. M.; Morges, M. A.; Rose, B. J.; Thamm, D. H.; Rovis, T. J. Am. Chem. Soc. 2012, 134, 13554.

22. (a) Feng, X.; Yun, J. Chem Commun (Camb) 2009, 6577. (b) Isegawa, M.; Sameera, W. M. C.; Sharma, A. K.; Kitanosono, T.; Kato, M.; Kobayashi, S.; Morokuma, K. ACS Catal. 2017, 7, 5370. (c) Iwai, T.; Akiyama, Y.; Sawamura, M. Tetrahedron: Asymmetry 2013, 24, 729. (d) Kitanosono, T.; Kobayashi, S. Asian. J. Org. Chem 2013, 2, 961. (e) Kitanosono, T.; Xu, P.; Kobayashi, S. Chem Commun (Camb) 2013, 49, 8184. (f) Kitanosono, T.; Xu, P.; Kobayashi, S. Chem Asian J 2014, 9, 179. (g) Kobayashi, S.; Xu, P.; Endo, T.; Ueno, M.; Kitanosono, T. Angew. Chem. Int. Ed. Engl. 2012, 51, 12763. (h) Radomkit, S.; Hoveyda, A. H. Angew. Chem. Int. Ed. Engl. 2014, 53, 3387. (i) Wang, L.; Chen, Z.; Ma, M.; Duan, W.; Song, C.; Ma, Y. Org Biomol Chem 2015, 13, 10691. (j) Wu, H.; Radomkit, S.; O'Brien, J. M.; Hoveyda, A. H. J. Am. Chem. Soc. 2012, 134, 8277. (k) Xie, J.-B.; Lin, S.; Luo, J.; Wu, J.; Winn, T. R.; Li, G. Organic Chemistry Frontiers 2015, 2, 42. (1) Zhu, L.; Kitanosono, T.; Xu, P.; Kobayashi, S. Chem. Commun. 2015, 51, 11685 .

23. Simpson, J. H.; Godfrey, J.; Fox, R.; Kotnis, A.; Kacsur, D.; Hamm, J.; Totelben, M.; Rosso, V.; Mueller, R.; Delaney, E.; Deshpande, R. P. Tetrahedron: Asymmetry 2003, 14, 3569.

24. Jankowski, P.; Marczak, S.; Wicha, J. Tetrahedron 1998, 54, 12071.

25. (a) Daniewski, A. R.; Kiegiel, J. J. Org. Chem. 1988, 53, 5534. (b) Fernandez, B.; Martinez Perez, J. A.; Granja, J. R.; Castedo, L.; Mourino, A. J. Org. Chem. 1992, 57, 3173. (c) Takahashi, T.; Naito, Y.; Tsuji, J. J. Am. Chem. Soc. 1981, 103, 5261. (d) Baggiolini, E. G.; Iacobelli, J. A.; Hennessy, B. M.; Uskokovic, M. R. J. Am. Chem. Soc. 1982, 104, 2945.

26. Wilson, S. R.; Haque, M. S. J. Org. Chem. 1982, 47, 5411.

27. Johnson, W. S.; Elliott, J. D.; Hanson, G. J. Am. Chem. Soc. 1984, 106, 1138.

28. López-Pérez, B.; Maestro, M. A.; Mouriño, A. Chem. Commun. 2017, 53, 8144.

29. Takahashi, T.; Tomida, S.; Doi, T. Synlett 1999, 1999, 644.

30. (a) Stork, G.; Hutchinson, D.; Okabe, M.; Parker, D.; Ra, C.; Ribereau, R.; Suzuki, T.; Zebovitz, T. Pure Appl. Chem. 1992, 64, 1809. (b) Stork, G.; Kobayashi, Y.; Suzuki, T.; Zhao, K. J. Am. Chem. Soc. 1990, 112, 1661. 
31. Sabroe, T. P.; Calverley, M. J. US 8759555 B2, Jun. 24, 2014.

32. (a) George, D. T.; Kuenstner, E. J.; Pronin, S. V. J. Am. Chem. Soc. 2015, 137, 15410. (b) Magnus, P.; Waring, M. J.; Scott, D. A. Tetrahedron Lett. 2000, 41, 9731. (c) Ishikawa, H.; Colby, D. A.; Seto, S.; Va, P.; Tam, A.; Kakei, H.; Rayl, T. J.; Hwang, I.; Boger, D. L. J. Am. Chem. Soc. 2009, 131, 4904. (d) Barker, T. J.; Boger, D. L. J. Am. Chem. Soc. 2012, 134, 13588. (e) Leggans, E. K.; Barker, T. J.; Duncan, K. K.; Boger, D. L. Org. Lett. 2012, 14, 1428. (f) Iwasaki, K.; Wan, K. K.; Oppedisano, A.; Crossley, S. W. M.; Shenvi, R. A. J. Am. Chem. Soc. 2014, 136, 1300. (g) Obradors, C.; Martinez, R. M.; Shenvi, R. A. J. Am. Chem. Soc. 2016, 138, 4962. (h) Yan, M.; Lo, J. C.; Edwards, J. T.; Baran, P. S. J. Am. Chem. Soc. 2016, 138, 12692. (i) Lo, J. C.; Kim, D.; Pan, C.-M.; Edwards, J. T.; Yabe, Y.; Gui, J.; Qin, T.; Gutiérrez, S.; Giacoboni, J.; Smith, M. W.; Holland, P. L.; Baran, P. S. J. Am. Chem. Soc. 2017, 139, 2484.

33. Chen, Z.-H.; Chen, Z.-M.; Zhang, Y.-Q.; Tu, Y.-Q.; Zhang, F.-M. J. Org. Chem. 2011, 76, 10173 .

34. Cox, C.; Danishefsky, S. J. Org. Lett. 2001, 3, 2899.

35. (a) Kolb, H. C.; VanNieuwenhze, M. S.; Sharpless, K. B. Chem. Rev. 1994, 94, 2483. (b) Walsh, P. J.; Sharpless, K. B. Synlett 1993, 1993, 605.

36. Bonazzi, S.; Binaghi, M.; Fellay, C.; Wach, J.-Y.; Gademann, K. Synthesis 2010, 2010, 631.

37. Parthasarathy, G.; Besnard, C.; Kündig, E. P. Chem. Commun. 2012, 48, 11241.

38. Haider, M.; Sennari, G.; Eggert, A.; Sarpong, R. J. Am. Chem. Soc. 2021, 143, 2710.

39. Defaut, B.; Parsons, T. B.; Spencer, N.; Male, L.; Kariuki, B. M.; Grainger, R. S. Org. Biomol. Chem. 2012, 10, 4926.

40. Kulesza, U.; Plum, L. A.; DeLuca, H. F.; Mouriño, A.; Sicinski, R. R. J. Med. Chem. 2015, 58, 6237.

41. (a) Cornella, J.; Edwards, J. T.; Qin, T.; Kawamura, S.; Wang, J.; Pan, C.-M.; Gianatassio, R.; Schmidt, M.; Eastgate, M. D.; Baran, P. S. J. Am. Chem. Soc. 2016, 138, 2174. (b) Edwards, J. T.; Merchant, R. R.; McClymont, K. S.; Knouse, K. W.; Qin, T.; Malins, L. R.; Vokits, B.; Shaw, S. A.; Bao, D.-H.; Wei, F.-L.; Zhou, T.; Eastgate, M. D.; Baran, P. S. Nature 2017, 545, 213. (c) Qin, T.; Cornella, J.; Li, C.; Malins Lara, R.; Edwards Jacob, T.; Kawamura, S.; Maxwell Brad, D.; Eastgate Martin, D.; Baran Phil, S. Science 2016, 352, 801. (d) Sandfort, F.; O'Neill, M. J.; Cornella, J.; Wimmer, L.; Baran, P. S. Angew. Chem. Int. Ed. 2017, 56, 3319. (e) Smith, J. M.; Qin, T.; Merchant, R. R.; Edwards, J. T.; Malins, L. R.; Liu, Z.; Che, G.; Shen, Z.; Shaw, S. A.; Eastgate, M. D.; Baran, P. S. Angew. Chem. Int. Ed. 2017, 56, 11906. (f) Toriyama, F.; Cornella, J.; Wimmer, L.; Chen, T.-G.; Dixon, D. D.; Creech, G.; Baran, P. S. J. Am. Chem. Soc. 2016, 138, 11132.

42. Blauvelt, A.; Chiricozzi, A. Clinic Rev Allerg Immunol 2018, 55, 379. 